\section{Anal cancer - impact of interstitial brachytherapy}

\author{
Kubecová $\mathrm{M}^{1}$, Vránová $\mathrm{J}^{2}$, Šejdová $\mathrm{M}^{1}$ and Regináčová $\mathrm{K}^{1 *}$ \\ 1'Department of Radiotherapy and Oncology, 3rd Faculty of Medicine, Charles University and University \\ Hospital Královské Vinohrady, Prague, Czech Republic \\ ${ }^{2}$ Department of Medical Biophysics and Medical Informatics. 3rd Faculty of Medicine, Charles University, \\ Prague, Czech Republic
}

\section{Summary}

We evaluated a total of 115 patients diagnosed with anal cancer, who were treated at our clinic from 1995 to 2012. Their average age was 61 years, most often were diagnosed in stages II and III, in most cases it was a squamous cell carcinoma located in the anal canal. The mean follow-up was 83 months (minimum 1 month and maximum 240 months). We combined external radiotherapy with boost of brachytherapy or boost of external radiotherapy and possibly a combination of both boosts. Half of the patients received concomitant chemotherapy. We specifically evaluated local tumor regression, overall survival and the impact to therapeutic effect of the chosen irradiation technique. Complete regression was achieved in 92 patients, partial regression in 21 patients. Overall survival, regardless of stage, was $80 \% 3$-year, 74\% 5-year and $67 \% 10$-year. The age of patients, the size of their own primary tumor and the therapeutic method used had a statistically significant effect on survival - especially the importance of brachytherapy was irreplaceable.

\section{More Information}

*Address for Correspondence: Klaudia Reginacova, Department of Radiotherapy and Oncology Faculty Hospital, Prague 10, Srobarova 50, 100 34, Czech Republic, Tel: +420 26716 3195; Email: klaudia.reginacova@fnkv.cz

Submitted: January 18, 2021 Approved: January 29, 2021 Published: February 01, 2021

How to cite this article: Kubecová M, Vránová J, Šejdová M, Regináčová K. Anal cancer - impact of interstitial brachytherapy. J Radiol Oncol. 2021; 5: 001-004.

DOI: 10.29328/journal.jro.1001034

Copyright: @ 2021 Kubecová M, et al. This is an open access article distributed under the Creative Commons Attribution License, which permits unrestricted use, distribution, and reproduction in any medium, provided the original work is properly cited.

Keywords: Anal cancer; Radiotherapy; Brachytherapy

\section{Check for updates}

OPEN ACCESS

\section{Introduction}

Anal cancer is one of the rarer diseases of the gastrointestinal tract, accounting for 1-2 percent of tumours of them. While its incidence in the Czech Republic has been gradually increasing over time, mortality has been stagnating. A key risk factor for tumour ethiology is infection with sexually transmitted human papillomaviruses, especially oncogenic HPV16 and 18. The prevalence in the population is significant and infection is detected in up to $80 \%$ of patients with anal cancer. Other risk factors include immunosuppression of the patient for any reason, and the effect of smoking has been proven. The most common histological type is a squamous cell carcinoma. Other variants are less common to rare. The issue of anal cancer is current and serious, especially because it is proven to be associated with sexually transmitted infections. There is latency in the order of decades from infection with oncogenic viruses to cancer outbreaks can be assumed. A reduction in incidence can be expected in the future due to the expansion of HPV vaccination. Before this happens, it is necessary to focus on improving the available treatment.

\section{Therapy}

Surgical treatment is reserved for small superficial lesions. Abdominoperineal resection is suitable for tumours that do not respond well to conservative treatment - tumour persistence or for local recurrence of the underlying disease. Radiotherapy is the method of choice for most anal cancers. Separate radiotherapy without potentiation of chemotherapy is reserved for T1 tumours. In other stages, the combination of external beam radiotherapy and concomitant chemotherapy is standard. Residual tumour, depending on its location and size, is irradiated by boost of externally radiotherapy or interstitial brachytherapy. The presented study aimed to compare different radiotherapy regimens used in the therapy protocol of $\mathrm{AC}$ in retrospective manner.

The subject of our work was, in addition to the evaluation of treatment results, to find out which factors significantly affect the results and whether the method of therapy used affects overall survival.

\section{Patient file}

Between 1995 and 2012, we treated a total of 134 patients with anal cancer at our clinic and 115 patients were evaluated for whom complete documentation was available. There were 89 women and 26 men. The average age was 61 years (ranging from 33 to 90 years). The squamous cell carcinoma predominated (101 patients), followed by 13 basaloid carcinomas and 1 apocrine carcinoma. Grading was not determined in 32 patients, G1 was in 14, G2 in 34, G3 in 32 and G4 in 3. 
Stage II (68 patients), stage III (29 patients), stage I (11 patients), stage IV (5 patients) was the most represented, and no stage was determined in two patients. The actual tumor size was expressed by the symbol T (TNM classification) - T1 (12), T2 (53), T3 (36), T4 (7), Tx (7). In most cases, the tumor was located in the anal canal, event. Spread from the canal to the marginal zone on the skin. Only 5 patients had marginal carcinoma alone, which did not spread to the canal.

All patients underwent external radiotherapy, in 10 cases to the pelvic area, the other 105 patients to the pelvic area and inguinal nodes at a total dose of 45 -50Gy, per fraction 1.8Gy.

Boost for residual tumor by interstitial brachytherapy alone was applied to 43 patients. It was a single-plane puncture using a circular template with a dose of 5-6 Gy Ir192 HDR for a reference isodose in $5 \mathrm{~mm}$ - a total of 2 fractions at an interval of 1 week. We applied brachytherapy most often 2 weeks after the end of external radiotherapy.

In 69 cases, the boost was combined - most often with an external dose of $10 \mathrm{~Gy}$ and then in 2 weeks with interstitial brachytherapy in 1 fraction. In 3 patients, the boost was applied intracavitary (intraanally) using a roller. It was a completely superficial minimal tumour residuum. The dose was applied to the surface of the cylinder ( $5 \times 2 \mathrm{~Gy}, 6 \times 2.5$ Gy, 7x3 Gy).

The total average duration of radiotherapy was 60 days, and the total average dose of radiotherapy was $59 \mathrm{~Gy}$ (min. 30 Gy, max. 79 Gy).

A total of 65 patients underwent chemotherapy, namely the $1^{\text {st }}$ week of radiotherapy mostly with the combination of 5 -fluorouracil (continuous $1^{\text {st }}-5^{\text {th }}$ day) together with Mitomycin and the $5^{\text {th }}$ week of radiotherapy only 5 -fluorouracil without Mitomycin. In some patients, Cisplatin was administered weekly.

The mean follow-up was 83 months (min. 1, max. 240).

\section{Results}

Complete regression (CR) was achieved in 92 patients, partial regression (PR) in 21 patients. Overall survival regardless of stage was very good: 3 -year-old $80 \%$, 5 -year-old $74 \%, 10$-year-old $67 \%$. Survival in individual stages was not statistically significant, but there were significant differences (trend), $p=0.06$, Table 1 . The effect of primary tumor size (T) on survival was statistically significant, $p=0.028$, Table 2 . On the contrary, the effect of grading on survival (G) was not statistically significant, but there is a trend (G3 worst), $p=0.057$, Table 3 . The patient's age had a statistically significant effect on survival, $p=0.001$. Worse therapy outcomes were seen with increasing age.
The results were interesting depending on the type of therapy used. Chemotherapy had no effect on overall survival, $p=0.26$, statistically insignificant. 5-year survival with chemotherapy was $80 \%$, without chemotherapy $71.3 \%, 10$-year $67 \% \times 68 \%$. The total dose of radiotherapy administered had no statistically significant effect on overall survival, $p=0.07$, but there was a trend (better results were obtained with a higher dose). Total radiotherapy time had no effect on survival, $p=0.23$, interval between external RT and subsequent boost also had no effect on survival, $p=0.58$. The most statistically significant factor with the greatest influence on overall survival was the used technique of boost brachytherapy, $p<0.001$, Table 4 . The use of interstitial brachytherapy statistically significantly improved the treatment results $p<0.001$.

\section{Summary}

The age of patients, the size of their own primary tumor and the method of therapy used had a statistically significant effect on survival - the importance of brachytherapy was especially irreplaceable. We have shown some trend in some other factors, such as tumor grading, disease stage or dose rate. The application of the BRT was confirmed to be irreplaceable.

The results were processed using statistical methods (Kaplan-Meier, Cox. Regression analysis).

\begin{tabular}{|c|c|c|}
\hline Table 1 & \\
\hline Stage & 5-year survival & 10-year survival \\
\hline I & $100 \%$ & $80 \%$ \\
\hline II & $78 \%$ & $68 \%$ \\
\hline III & $69 \%$ & $56 \%$ \\
\hline IV & $62 \%$ (a few patients) & $62 \%$ (a few patients) \\
\hline & & $p=0.06$ \\
\hline
\end{tabular}

\begin{tabular}{|c|c|c|}
\hline Table 2 & \multicolumn{2}{|c|}{} \\
\hline Tumour & 5-year survival & 10-year survival \\
\hline T1 & $100 \%$ & $80 \%$ \\
\hline T2 & $82 \%$ & $75 \%$ \\
\hline T3 & $55 \%$ & $42 \%$ \\
\hline T4 & $62 \%$ (a few patients) & $62 \%$ (a few patients) \\
\hline & & $p=0.028$ \\
\hline
\end{tabular}

\begin{tabular}{|c|c|c|}
\hline Table 3 & \multicolumn{2}{|c|}{} \\
\hline Grading & 5-year survival & 10-year survival \\
\hline 1 & $85 \%$ & $69 \%$ \\
\hline 2 & $75 \%$ & $70 \%$ \\
\hline 3 & $68 \%$ & $57 \%$ \\
\hline & & $p=0.057$ \\
\hline
\end{tabular}

\begin{tabular}{|c|c|c|c|}
\hline \multicolumn{4}{|l|}{ Table 4} \\
\hline Boost & 3-year survival & 5-year survival & 10-year survival \\
\hline interstitial & $95 \%$ & $92 \%$ & $80 \%$ \\
\hline Interstitial + external & $93 \%$ & $71 \%$ & $57 \%$ \\
\hline External & $55 \%$ & $52 \%$ & $47 \%$ \\
\hline & & & $p<0.001$ \\
\hline
\end{tabular}




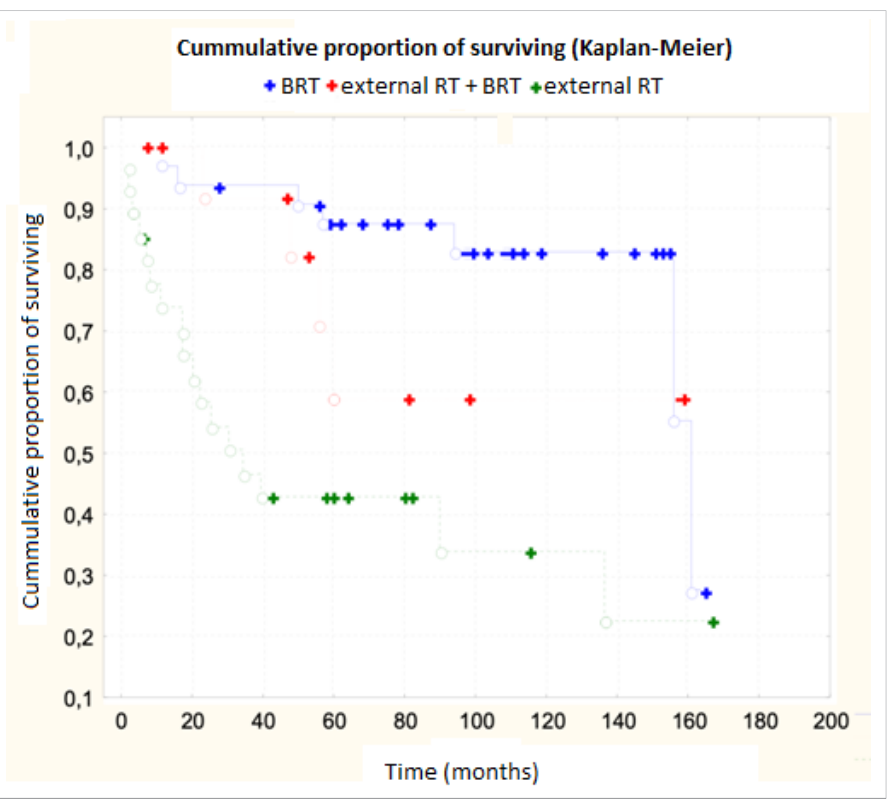

\section{Discussion}

Our experience shows the importance of using brachytherapy in local disease control and overall survival. There are not many workplaces with experience with brachytherapy as a part of treatment and they also differ in the method used.

A retrospective analysis from 2016 Gryc, et al. analyzed 190 patients with anal cancer, of whom 47 did not achieve complete remission of the disease after external chemoradiotherapy. These patients underwent boost interstitial brachytherapy 6 weeks after the end of external beam radiotherapy. A 5-year recurrence of the disease was observed in $24 \%$ of patients with a BRT boost and $19 \%$ without a brachytherapy boost ( $p=0.238)$. The 5 -year DFS, OS, and colostomy free interval were $74 \%, 75 \%$, and $76.1 \%$ in the BRT group and 69\% ( $p=0.212), 72 \%(0.924)$, and $82.7 \%$ ( $p=0.488)$, respectively without it. No differences in late toxicity were observed. The group of patients shows the importance of adding brachytherapy with an increased dose of Dmean 67.5 Gy to improve disease control without exacerbating late side effects [1].

Another retrospective analysis of the German group Heilman, et al. following 52 patients between 2000 and 2017 shows local disease control in $93 \%$ of patients indicated for boost of brachytherapy. Patients underwent a combination of external RT to ld. 46Gy and concomitant chemotherapy. Boost image-guided brachytherapy was applied using PDR to a total dose of 60Gy (46-65Gy). $60 \%$ of patients had stage $\mathrm{T} 2$ and $29 \%$ of patients had infiltrated nodes. Patients were followed for an average of 36.6 months (30-105). In the study group, overall survival was $83 \%$ and $91 \%$ of patients had no colostomy. Of the long-term serious effects, necrosis was observed in $4 \%$ and incontinence in $13 \%$ [2].
A retrospective meta-analysis of Frakelli, et al. from 2018 focused on work in which the median follow-up was 5 years, patients were treated with chemoradiotherapy. They focused on LC/LRC, DFS, OS and the proportion of patients without colostomy. They evaluated a total of 10 works. LC/LRC was 78.6\% (70.7 - 92), DFS 75.8\% (65.9-85.7), OS 69.4\% (63.4-82) and no colostomy $76.1 \%$ (61.4 - 86.4). The unambiguous importance of brachytherapy has not been demonstrated. However, the work points to the need for a study comparing the results of treatment of patients treated with boosted brachytherapy vs. boost of external radiotherapy vs. without boost [3].

Further work comparing boost by interstitial brachytherapy and external boost shows comparable treatment results. The work retrospectively analyzed 81 patients treated with external radiotherapy to ld.45Gy with concomitant chemotherapy with mitomycin and 5-FU. Thereafter, patients subsequently underwent an external boost of $14.4 \mathrm{~Gy} / 7$ fractions or with an interval of interstitial brachytherapy of $14 \mathrm{~Gy} / 7$ fractions at 3 weeks. Complete remission of the disease occurred in $93.4 \%$ of patients. At low stages, 5-year local disease control was 100\% using brachytherapy. In the group of all patients, the use of external boost or brachytherapy boost led to comparable results. 5-year and 10-year survival was $66 \%$ and $44 \%$ in the brachytherapy group and $66 \%$ and $52 \%$ in the external RT group. Acute toxicity of treatment was better in the group with brachytherapy ( $p=0.14$, stage I-IIIa $p=0.005)$, late toxicity was comparable [4].

Node involvement does not appear to be a contraindication to the use of brachytherapy. A retrospective analysis of the CORS-03 study followed 229 patients between 2000 and 2005, of whom 99 had nodal involvement - N1 67 patients and N2/3 32 patients. Patients underwent external RT and then boosted external RT or brachytherapy. With satisfactory tumor regression, brachytherapy was also important in N1 [5].

In a retrospective analysis of 209 patients followed from 1992 to 2007, it also shows the importance of brachytherapy. Of the cohort, 163 were stage II and IIIA patients and 53 were N1-3. Patients were treated according to general condition and comorbidities with alone external beam radiotherapy (58) or chemoradiotherapy (151), all patients had boost brachytherapy. The median follow-up was 72.8 months. The 5- and 10-year local disease control was 78.6 and $73.9 \%$, respectively. Acute and late toxicity of G3-4 occurred in 11.2 and $6.3 \%$ of patients. Only $6 \%$ of patients ended up with a permanent stoma due to severe toxicity [6].

In the evaluation of the results of the method used, the time of evaluation of the treatment result is a great importance in 
anal cancer. This is confirmed by the work of Glynne-Jones, et al. published in 2017 in the Lancet, which followed a total of 960 patients treated between 2000 and 2007. Many patients regressed within 26 weeks of starting treatment [7].

Equally important are side effects. The use of IMRT in radiotherapy planning reduces acute toxicity in patients receiving chemoradiotherapy. In contrast, the combination of chemoradiotherapy and boost brachytherapy reduces late toxicity [8].

Individual retrospective analyzes are not consistent, they use different techniques of brachytherapy, some HDR, some PDR. In the future, a prospective study would be appropriate to confirm the importance of boost brachytherapy or boost external radiotherapy for anal cancer.

\section{Conclusion}

Anal cancer is one of the most treatable and highly treatable cancers. It is therefore necessary to always choose a suitable treatment modality and apply a sufficient dose of radiation correctly. External radiotherapy using the IMRT technique is the method of choice, it is accurate and gentle. Boost brachytherapy is important especially in the low stages of the disease (the tumor must not extend half of the circumference of the anal canal to avoid impaired sphincter function). In our cohort, we demonstrated the undeniable importance of boost brachytherapy even with an effect on overall survival.

\section{References}

1. Gryc $T$, Ott O, Putz F, Knippen S, Raptis D, et al. Interstitial brachytherapy as a boost to patients with anal carcinoma and poor response to chemoradiation: Single-institution long-term results. Brachytherapy. 2016; 15: 865-872.

PubMed: https://pubmed.ncbi.nlm.nih.gov/27720203/

2. Heilmann. Poster - Image guided boost brachytherapy for anal cancer: tumor and functional outcome, 2018, Radiotherapy and Oncology. 2018. https://www.postersessiononline.eu/173580348_eu/ congresos/ESTRO37/aula/-V_252_ESTRO37.pdf

3. Frakulli R, Buwenge M, Cammelli S, Macchia G, Farina E, et al Brachytherapy boost after chemoradiation in anal cancer: a systematic review. J Contemp Brachytherapy. 2018; 10: 246-253.

PubMed: https://www.ncbi.nlm.nih.gov/pmc/articles/PMC6052386/

4. Oehler-Jänne C, Seifert B, Lütolf UM, Studer G, Glanzmann C, et al. Clinical outcome after treatment with a brachytherapy boost versus external beam boost for anal carcinoma. Brachytherapy. 2007b; 6 : 218-226.

5. Moureau-ZabottoL, Ortholan C, Hannoun-LeviJM, TeissierE, CowenD, et al. Role of Brachytherapy in the Boost Management of Anal Carcinoma With Node Involvement (CORS-03 Study). Int J Radiat Oncol. 2013b; 85: e135-e142.

6. Lestrade L, De Bari B, Pommier P, Montbarbon X, Lavergne E, et al. Role of brachytherapy in the treatment of cancers of the anal canal. Strahlentherapie Und Onkologie. 2014; 190: 546-554.

7. Carvalho C, Glynne-Jones R. Challenges behind proving efficacy of adjuvant chemotherapy after preoperative chemoradiation for rectal cancer. Lancet Oncol. 2017; 18: e354-e363. PubMed: https://pubmed.ncbi.nlm.nih.gov/28593861/

8. Saarilahti K, Arponen P, Vaalavirta L, Tenhunen M. The effect of intensity-modulated radiotherapy and high dose rate brachytherapy on acute and late radiotherapy-related adverse events following chemoradiotherapy of anal cancer. Radiother Oncol. 2008; 87:383-390. PubMed: https://pubmed.ncbi.nlm.nih.gov/18501454/ 